\title{
Sobre el sentido y la utilidad de la Historia de la Medicina (1895-1970)
}

\author{
Enrique Laval Manriquet
}

\section{On the meaning and usefulness of History of Medicine (1895-1970)}

The knowledge of the history of Medicine does not seem to add "utility" to the activities of a physician. Nevertheless, for a doctor eager to get good intellectual information -or the spiritual perfection called "culture"- the autor gives some reasons on why being acquainted with the history of medicine has some "utility" through the following items: Intellectual dignity: by deliberately and gratefully assuming the best of those who introduced technical devices or methods, as auscultation, or intellectual elaborations, like the etiologic model of Galen. Moral clarity: to perform as if they were his own, great deeds of the personages whose discoveries built the concepts and procedures used nowadays. Intellectual clarity: assuming the reason for being and the sense of what is being done. Intellectual liberty: knowing history in a comprehensive way, frees a doctor from the temptation of considering scientific notions as unmistakably true and definite. A possible option to originality: stimulated by a will of emulation, to search for less insecure or more rewarding knowledge, tending to "complete" what is at present accepted.

Key words: History medicine, utility, physician, culture.

Palabras clave: Historia, medicina, utilidad, médico, cultura.

$\mathrm{T}$ ema viejo y constante en la cultura occidental, éste de la utilidad y el sentido del conocimiento de la historia. Desde Tucídides y Cicerón, en el mundo antiguo, hasta Nietzsche, Valéry y Ortega, en el más inmediato ayer, todas las opiniones han sido vertidas; desde aquellas que hacen del conocimiento histórico una suerte de férula para el buen crecimiento espiritual de los hombres y los pueblos -el ciceronismo Historia vitae magistra hasta las de quienes, seducidos por una encandilada estimación del puro presente - tal la del "presencialista" Valéry-declaran ser puro y deletéreo narcisismo mental la exploración del pasado.

Más o menos directa y expresamente influida por una de esas opiniones, la que en todo el Occidente difundió el positivismo de Augusto Comte, los médicos de los siglos XIX y XX solieron mirar con despego, cuando no con irónica aversión, el conocimiento de la historia de su propia disciplina. Entre los intelectualmente menos ambiciosos, los puros técnicos repetidores de técnicas que otros inventaron, porque el saber histórico no parece añadir perfección alguna a la ejecución correcta de las operaciones manuales, instrumentales y mentales - la percusión, la auscultación, la inducción diferencial, la gastrectomía, etc. - en que de ordinario consiste la práctica del diagnóstico y del tratamiento. $\mathrm{Y}$ entre aquellos cuya ambición intelectual es patente -los profesores bien informados, los investigadores-, porque ha prevalecido la idea de que la obra de la inteligencia humana fue puro mito o inútil construcción a priori, antes de que las mentes

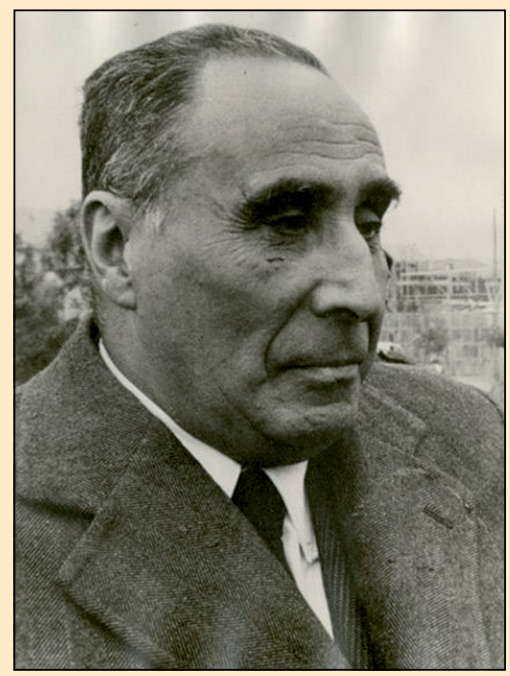

de los hombres de ciencia, indeliberadamente en unos casos, consciente y reflexivamente en otros, llegaran a ser guiadas por los principios y las orientaciones del método experimental y positivo. "Historia, ¿para qué?" tal es la interrogación tópica en la actitud o en los labios de los médicos que a sí mismos se consideran "serios y científicos". El saber histórico que a estos importa no rebasaría los límites del que constituye la bibliografía que suele acompañar a las publicaciones de las revistas científicas; a lo sumo, los dos o tres decenios anteriores a la confección
Recibido: 13 de julio de 2017

Correspondencia a: Enrique Laval R. revinf@sochinf.cl 
del trabajo en cuestión. Lo demás, lo que acerca de ese tema o de otros más o menos próximos hayan podido decir Hipócrates, Galeno, Avicena, Paracelso, e incluso Sydenham, Boerhaave o Albrecht von Haller, no pasaría de ser vaga y amena literatura, buena, en el mejor de los casos, para lucimiento de quien sea capaz de exhibirla a la hora "poco seria" de la sobremesa o de la tertulia.

¿Es así? La sentencia de "inutilidad" - acaso la más grave de cuantas puede formular el judicial espíritu del hombre moderno - ¿deberá caer resuelta e inexorablemente sobre el conocimiento de la historia de la Medicina? Quienes académica o vocacionalmente hacen de esta disciplina la ocupación principal de su vida, ¿habrán de resignarse a vivir en el mundo científico de trop pour l'éternité, para decirlo con la tan difundida expresión sartriana?

Como en todas sus tareas, la inteligencia responsable debe comenzar, frente a ésta, distinguiendo los diversos sentidos del término sobre qué debe pronunciarse; en nuestro caso, los diversos sentidos posibles del término "utilidad". Si sólo se reputa "útil” lo que proporciona constante y sonante lucro material o concreta habilidad técnica, habrá que afirmar sin reservas que ese juicio negativo respecto del saber histórico del terapeuta tiene, como suele decirse, toda la razón. Para quien considere el ejercicio de la Medicina sólo como una profesión más o menos lucrativa o como la mera ejecución habitual y más o menos correcta de técnicas diagnósticas y terapéuticas perfectamente regladas, la historia del saber médico será, sin el menor género de duda, pura inutilidad. ¿Podrá pensar lo mismo el médico - más genéricamente: el hombre - que en cuanto médico y en cuanto hombre aspire a poseer en mayor o menor medida esa perfección del espíritu a que solemos dar el amplio y expresivo nombre de "cultura"? Independientemente de la fruición que su lectura pueda producir, ¿no es cierto que un diálogo de Platón, el Quijote y un poema de Antonio Machado poseen alguna "utilidad" para quien se proponga ser, además de buen técnico, "hombre culto"? ¿No es cierto que esas lecturas son "útiles", valga la expresión, para levantar el nivel de la condición humana o, si se quiere, para hacer que el hombre sea "más hombre?, más perfecto poseedor de ese genérico y personal modo de ser que los hispanohablantes designamos con el término de "hombredad"?

Pues bien: para quienes como médicos no se contenten con ser simples "técnicos repetidores de técnicas", para cuantos aspiren a poseer en su persona la doble perfección del "hombre culto" y del "técnico intelectualmente ambicioso" - con otras palabras: para los médicos deseosos de buena información intelectual o francamente volcados a la investigación-, he aquí el elenco de las principales razones por las cuales posee alguna "utilidad" el conocimiento de la historia de la Medicina:
I. Ese conocimiento otorga, en primer término, dignidad intelectual. No parece ilícito afirmar que la formación histórica en el campo de su propia disciplina concede al médico, en cuanto tal médico, esa a la vez básica y cimera nobleza que la lengua popular castellana suele llamar "buena nacencia" o condición de "hombre bien nacido". ¿Quién es, en efecto, bien nacido? No simplemente el que tiene origen noble sino, de modo más amplio y hondo, quien deliberada, consciente y agradecidamente quiere y sabe asumir en su propia vida lo mejor de cuanto hubo en las de aquellos de quienes como hombre procede.

Imaginemos el caso de un buen técnico de la auscultación. Si ese médico, además de saber auscultar, sabe con agradecimiento quien fue Laennec, y qué hizo para inventar la auscultación mediata, y por qué y cómo hizo eso que hizo, se elevará sólo con esto a la decorosa condición de médico y hombre "bien nacido". Procediendo de otro modo será un rudo logrero de la técnica, un sujeto que explota en beneficio suyo la decisiva invención. De Laennec sin haber tributado a éste el mínimo homenaje a que por su preciosa hazaña científica tiene derecho: ese homenaje que rinden, respecto del creador de algo, quienes se acerca a él con voluntad de conocimiento y de reconocimiento. Y lo que se dice de una invención técnica - llámese a ésta termometría clínica, auscultación mediata o seroaglutinación, con el mismo derecho puede y debe decirse de las invenciones intelectuales que la historia ha revelado ser orientadoras y fecundas: por ejemplo, el esquema etiológico de Galeno, el concepto de "especie morbosa" o la anatomía comparada de los morfólogos "idealistas".

II. La frecuentación de la historia de la Medicina regala al médico, por otra parte, claridad intelectual, y hasta "claridad" a secas, esa a que los viejos escritores de nuestra lengua aluden cuando dan a un hombre el título de "claro varón". En efecto: desde un punto de vista moral, y a éste es al que suele referirse la expresión castiza, la claridad del "claro varón" consiste en ejecutar, viéndolas como propias, hazañas a la vez ejemplares y brillantes. Conviene no olvidar, sin embargo, que, junto a ésta de carácter moral, más aún, dentro de ella, hay y tiene que haber siempre, como parte integral suya, una claridad de índole intelectual, la que discierne el hecho de conocer y poseer lúcidamente la razón de ser y el sentido de aquello que se hace, así en orden a la vida personal como desde el punto de vista de la vida humana in genere. ¿Podría darse acaso el título de "claro varón" al inconsciente y atolondrado, por esforzadas que parecieran ser las hazañas por él cumplidas?

Apliquemos estas esquemáticas ideas al caso del médico, mediante la consideración de un ejemplo trivial. Diagnosticar correctamente una estenosis mitral puede ser en 
ocasiones una módica hazaña asistencial y técnica. Pues bien: tal hazaña convertirá en "claro varón” al médico que la cumple, concederá a éste claridad intelectual, cuando sea realizada con un conocimiento cierto y preciso de lo que históricamente es y significa la mentalidad anatomoclínica; esto es, la empresa histórica que sucesivamente han llevado a término - valgan como hitos indicadores estos pocos nombres - Lancisi, Albertini, Morgagni, Bichat, Corvisart, Bayle, Laennec y Bright. Ejecutar con alguna perfección un empeño técnico cualquiera es, a la postre, resolver personalmente un problema intelectual: y la claridad que de suyo irradia el hecho de resolverlo, llega a su máximo cuando se conoce con rigor la historia de ese problema. He aquí, pues, una tarea ineludible de los historiadores de la Medicina, si estos quieren que su disciplina sea considerada como "útil" por parte de los médicos intelectualmente ambiciosos: construir y exponer sugestivamente la historia de los problemas fundamentales del arte de curar, desde los morfológicos y fisiológicos hasta los médico-sociales.

III. Tercer fruto principal del saber histórico, sea o no sea médico su carácter, es la libertad intelectual. Conocer la historia de un modo comprensivo y no puramente memorístico da libertad a la mente respecto aquello que se sabe, y tal es una de las más profundas razones que justifican el cultivo de ese conocimiento.

¿Acaso no es así? Hay, en efecto, saberes "obligantes": son aquellos en los cuales la mente humana se siente forzada al aserto en que ellos consisten. Saber, por ejemplo, que dos más dos son cuatro, es verse obligado necesariamente a pasar del primero al segundo de los términos de esa sencilla igualdad. Con las restricciones que Boutroux impondría - recuérdese su célebre memoria sobre "la contingencia de las leyes de la naturaleza", lo mismo cabría decir de la ley de caída de los graves y de las ecuaciones del campo electromagnético.

Pero junto a estos saberes - y en cierto modo sobre ellos - existen otros en el globus intellectualis del espíritu humano, a los que conviene el nombre de saberes "liberadores". Son aquellos en los cuales la mente se siente liberada de aceptar como necesaria la proposición de aquello que se sabe; y apenas será necesario decir que entre ellos son precisamente los de carácter histórico los que más clara y enérgicamente cumplen esta sutil faena de liberación espiritual.

Quien sin formación ni perspectiva histórica aprende las nociones científicas correspondientes a la situación en que él existe y se educa, con gran facilidad caerá en la tentación de considerarlas incuestionablemente ciertas y definitivas. Pero si ha sido enseñado a contemplar cómo las "teorías" y los sistemas nacen y pasan en el curso de la historia, aprenderá a aceptar sin dogmatismo, y por lo tanto con íntima libertad intelectual, lo que de ninguna manera puede ser elevado a la condición de dogma. La historia, el saber histórico, es el recurso supremo para conseguir aquel sano "antidogmatismo" que tan oportuna y certeramente propuso a los médicos Gregorio Marañón como antídoto contra la tiranía de los "sistemas".

No se postula con ello un relativismo gnoseológico radical; no se afirma que la mente del hombre no puede conquistar nociones, principios o axiomas de validez inmutable. Pero sí se evita, y no es poco, la atribución de un carácter obligante y definitivo a las construcciones científicas - o seudocientíficas - de los doctrinarios de la Medicina, llámense tales construcciones "brownismo", como a fines del siglo XVIII y comienzos del XIX, "virchowismo", como en la segunda mitad del XIX, o "teoría reflexológica" como en los decenios centrales del siglo $\mathrm{XX}$.

El médico sin formación histórica se hallará a veces en grave riesgo de formular, frente a un aspecto cualquiera de la realidad, una de las varias expresiones con que la mente del hombre manifiesta la peligrosa tentación hacia la tiranía lógica que en ella late: "Esto no es más que...", "O esto, o lo otro", u otras semejantes.

Frente a los dogmáticos y a los sistematizadores a ultranza sólo cuando incurren en pretensión del dogmatismo son condenables el esprit de système y el esprit systèmatique. Tendencias ineludibles del espíritu humano-, el hombre educado en la recta comprensión de la historia dirá siempre, en cambio, con libertad y con razón, las tan repetidas y salvadoras palabras de Hamlet: "Hay más cosas en los cielos y en la tierra, Horacio, de las que enseña tu filosofía”.

IV. La formación histórica concede al hombre de ciencia, por último, cierta opción a la originalidad. Sólo el hecho de mostrarle el carácter provisional y movedizo del suelo histórico que pisa - con otras palabras: sólo esa libertad intelectual a que el apartado anterior se refiere, moverán frecuentemente al hombre de ciencia, médico o no, a buscar saberes menos inseguros o más íntimamente satisfactorios que aquellos cuya validez duda. Mas no sólo por esta vía es capaz de incitar hacia la originalidad el saber histórico. Junto a ella hay otras tres, que sumariamente conviene nombrar y describir.

La familiaridad con la historia de un saber suscita en el ánimo de quien la posee, siempre que este ánimo sea sensible y ambicioso, cierta voluntad de emulación. “¿Por qué yo - se pregunta entonces el hombre - no he de ser capaz de hacer por mí mismo y en mi situación lo que en la suya hicieron los protagonistas de la historia de esto que yo sé?" Así se encendió en el alma de Vesalio su decisión de rivalizar con Galeno en orden al saber anatómico, y aun de superarle, según lo que el gran anatomista bruselés cuenta de sí mismo en el prólogo de su Fabrica. Y con intención a la vez autobiográfica y educativa, eso mismo 
viene a decirnos Ramón y Cajal cuando en "Reglas y consejos para la investigación biológica" expone su idea de lo que debe ser la lectura de un texto innovador y magistral.

Menos “personal", en el sentido tópico de esta palabra, pero no menos eficaz que la voluntad de emulación antes mencionada, es el propósito de invención completiva que el conocimiento de la historia de un determinado saber despierta en las mentes alertadas y generosas. "Saber lo que hizo" -no otra cosa es, en esencia, el fruto de la información histórica- supone siempre, cuando la inteligencia no es meramente respectiva, saber o sospechar lo que pudo hacerse y no se hizo, porque nadie es capaz de agotar las posibilidades de creación original que su propia situación le brinda. Como anatomopatólogos de la inflamación -valga este ejemplo-, Virchow y Conheim hicieron mucho, pero no todo lo que ellos mismos hubieran podido hacer; por lo cual, leyendo con mente a la vez "científica" e "histórica" lo que uno y otro escribieron, no será improbable que en la mente del lector surja un proyecto de investigación original enderezado a "completar inventivamente" lo que Virchow y Conheim hicieron. Y lo que se dice de ellos, no será difícil aplicarlo a todos los posibles descubridores de hechos y creadores de teorías.

La lectura de los textos científicos del pasado conducirá a veces, en fin, al recuerdo de lo olvidado. Por extraño que parezca, los hombres olvidan con frecuencia hallazgos científicos importantes, aunque de ellos haya quedado constancia escrita; y cuando esto acontece, una indagación atenta de los textos del pasado permitirá conquistar a poca costa dignidad de "inventor". Suponiendo que entonces hubiese sido posible, la exploración de los manuscritos árabes del Cairo durante el siglo XV -entre ellos estaba el de Ibn-al-Nafis- que contiene la primera descripción de la circulación menor- habría permitido adentrarse en cien años, sólo por la vía de la investigación histórica, al descubrimiento que por el camino de la observación anatómica hizo más tarde Miguel Serveto. Otro tanto cabe decir de la auscultación inmediata del tórax, conocida sin duda alguna por los asclepíades hipocráticos, según el testimonio del escrito de morbis, y totalmente olvidada hasta que en los primeros años del siglo XIX Corvisart, Bayle y Laennec comienzan otra vez a practicarla. Ni siquiera es preciso ir tan lejos. Hoy, cuando la bibliografía de los trabajos que aparecen en las revistas científicas sólo suele referirse a los diez o quince años anteriores a la fecha de publicación, tengo por seguro que un examen minucioso de las colecciones de las revistas europeas y americanas publicadas entre 1870 y 1930 conducirá a "descubrir" hechos e ideas que desde entonces han desaparecido de la memoria de los médicos.

Dignidad, claridad, libertad y opción a la originalidad personal: tal es el balance de la "utilidad" de la historia de la Medicina, cuando seria y decorosamente se la estudia y cultiva. El recuerdo, dijo certera y donosamente Ortega y Gasset, es la carrerilla que el hombre se toma para saltar hacia adelante en la ejecución de sus proyectos. El hombre recuerda el pasado para lanzarse hacia el futuro. Ampliando al orden de la vida colectiva la validez indudable de esa sentencia, bien puede decirse que la historia -el saber histórico- es un recuerdo al servicio de una esperanza. Cuando más profunda y articulada, cuando menos gratuita y ligera sea la esperanza, tanto más hondo y pormenorizado habrá de ser el conocimiento del pasado. El hecho de ser médico no constituye una excepción a esa regla áurea de la existencia de los hombres en el tiempo.

\section{Resumen}

El conocimiento de la historia de la Medicina no parece aportar "utilidad" a la ejecución de las acciones del médico. No obstante, para el médico deseoso de buena información intelectual -o perfección del espíritu llamada "cultura"- el autor da algunas razones de por qué estar familiarizado con la historia de la Medicina tiene cierta "utilidad", mediante los siguientes ítems: Dignidad intelectual: por asumir deliberada y agradecidamente lo mejor de quienes aportaron elementos técnicos, como la auscultación, o elaboraciones intelectuales, como el esquema etiológico de Galeno. Claridad moral: ejecutar, viéndolas como propias, hazañas de la sucesión de personajes cuyos hallazgos construyeron los conceptos y procedimientos actualmente en uso. Claridad intelectual: poseer la razón de ser y el sentido de aquello que se hace. Libertad intelectual: conocer la historia, de un modo comprensivo, libera de la tentación de considerar las nociones científicas como incuestionablemente ciertas y definitivas. Cierta opción a la originalidad: estimulado por una voluntad de emulación, emprender la búsqueda de saberes menos inseguros o más satisfactorios, que tiendan a "completar" lo actualmente aceptado. 